\title{
El agua en la cuenca del río Jordán: la lucha por un recurso escaso ${ }^{1}$
}

\section{Ferran Izquierdo Brichs}

Becario de Investigación en Relaciones Internacionales

Centre d'Estudis sobre la Pau i el Desarmament

Universitat Autònoma de Barcelona. 08193 Bellaterra (Barcelona). Spain

\section{Resumen}

El agua ha sido un elemento importante en las relaciones entre átabes e istaelies, no sólo como causa de conflicto sino también por haber sido el factor central del único intento de cooperación entre los distintos Estados de la cuenca del Jordán. Así, los recursos hidrológicos en esta región adquieren una clara dimensión política, económica y de seguridad, incluso militar. El articulo aborda estas dimensiones de la problemática del agua a lo largo de la historia del conflicto árabe-istaelf.

Palabras clave: tecutsos hidrológicos, Jordán, conflicto árabe-istaelí, negociación Johnston, Oriente Medio.

\section{Abstract. Water in the Jordan basin: the fight for a scarce resource}

Freshwater has been one of the key elements in Arab-Israeli relations; not only as a cause for conflict but also for its having beten the main factor in the only attempt at cooperation among the different states in the Jotdan basin. Thus, water resources in the region attain clear political, economic, and - even military - security dimensions. The article tackles these dimensions of the water issue along with the history of the Arab-Israeli conflict.

Key words: water resources, Jordan, Arab-Israeli conflict, Johnston negotiation, Middle East.

\begin{aligned} & \multicolumn{2}{c}{ Sumario } \\ & Introducción Las negociaciones Johnston \\ & Hidrografla de la cuenca del río Jordán $(1953-1956) \\ &$ El inicio del conflicto De la gestión unilateral a la conquista \\ & 1949: la cuestión dal agua israelí del agta $(1955-1978) \\ &$ en la posguerra Apuntes sobre el presente \\ & Bibliografía citada \end{aligned}

1. Este attículo está basado en la memoria de licenciatura del autor, dirigida por Esther Barbé y presentada en la Facultat de Ciències Polficiques i de Sociologia de la UAB en septiembre de 1994. 


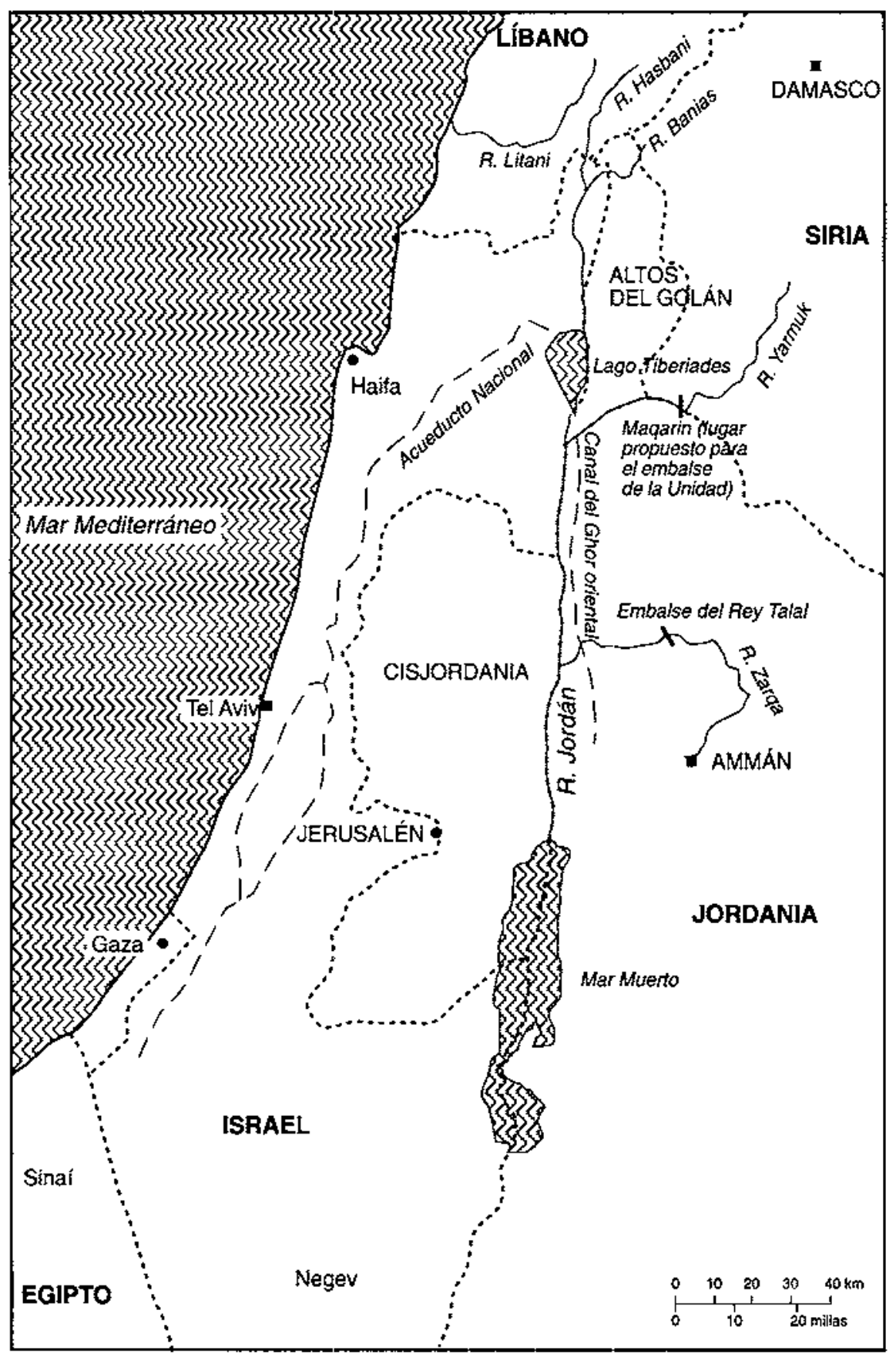

Figura 1. La cuenca del río Jordán. 
La próxima guerra en Oriente Medio se librará por el agua. Butros Ghali, 1985

La única cuestión que volverá a llevar a Jordania a la guerra es el agua

Rey Hussein de Jordania, 1990

\section{Introducción}

El agua es fuente de vida: unat afirmación banal por su evidencia pero que expresa una realidad que muchos hemos ignorado hasta hace pocos años, mientras que para otros constituía un elemento central para su desarrollo económico, social e, incluso, para su seguridad. Las zonas con carestía de agua, como la orilla sur del Mediterráneo y cada vez más también la orilla norte, encuentran un obstáculo añadido a sus necesidades de desarrollo, coincidiendo, en la mayoría de los casos, con un elevado índice de crecimiento demográfico, situación que la convierte en una cuestión de seguridad ecológica.

Actualmente 25 Estados sufren cortes crónicos de agua y las previsiones para principios del siglo XXI incrementan el númeto a 90 , la mitad de la población mundial, con las consecuencias conocidas de hambre y enfermedades. En el año 1992 había en el mundo 214 ríos y lagos compartidos ${ }^{2}$, algunos incluso por una docena de Estados: dos mil millones de personas dependían de la cooperación entre gobiernos para garantizar el suministro de recursos hidrológicos (Starr, 1992: 32). La dimensión internacional de la problemática del agua es cada día más clara y se puede manifestar en relaciones de conflicto o de cooperación.

Oriente Medio, una de las regiones más problemáticas del mundo, tiene en el agua una causa añadida de conflictos. A la carestía provocada por una geografia y climatología particularmente desfavorables, se suma el hecho de que sus principales rios son internacionales. El Nilo, el Eufrates y el Jordán son imprescindibles para las poblaciones de unos países - Egipto, Siria e Iraq, Israel y Jordania - en los cuales no caen las precipitaciones que alimentan estos ríos. Por ello, la gestión de los recursos hidrológicos se convierte en un problema de seguridad nacional para estos Estados.

La cuenca del Jordán, durante el siglo XX, se nos presenta como un modelo casi ideal de los conflictos que puede conllevar un recurso compartido. El agua, para judíos y árabes, es un bien precioso. Para los primeros fue imprescindible en el proceso de creación y consolidación del Estado de Israel, mientras que los árabes la necesitaban para la supervivencia y para evitar la colonización judía de la tietra de Palestina. Así, el agua se insertaba completamente en el marco político de la lucha entre los dos pueblos. Al convertirse en un problema de relaciones internacionales, los recursos hidrológicos en la zona de Palestina han generado diferentes niveles de contactos: desde el enfrenta-

2. Este número serfa mucho mayor si incluyéramos las aguas subterráneas. 
miento abierto al diálogo, y desde la dimensión de seguridad y "alta política» hasta los contactos para la gestión y administración de los recursos en un nivel de política usuave" (low politics).

El problema del agua puede convertirse en un elemento crucial de las negociaciones de paz que se están desarrollando en la zona ${ }^{3}$, tanto por su capacidad de generar nuevos conflictos como de obligar a las principales partes implicadas a cooperar en la gestión de los recursos hidrológicos. Así, el estudio de la evolución de la disputa por el agua puede ser útil para la comprensión del futuro de la cuenca del Jordán y Palestina.

\section{Hidrografia de la cuenca del río Jordán}

Una de las principales características del clima de esta zona es la rapidez con que cambian sus condiciones a medida que nos separamos de la costa, debido a las cordilleras montañosas y a los fuertes desniveles. En un área muy pequeña entran en contacto las influencias continentales con las mediterráneas $y$, a causa de la altitud y continuidad de las cordilleras, también hay un régimen especial montañoso de temperaturas más bajas y lluvias más abundantes. Las diferencias climáticas no sólo son pronunciadas de oeste a este, también aparecen de norte a sur: el viento dominante durante todo el año es de suroeste, por lo que en el norte es más húmedo ya que es de influencia marítima, mientras que en el sur es de influencia desertica (Fisher, 1978: 415, 416). La pluviosidad es muy baja excepto en la cordillera del monte Hermon (jabal ash-Sheikh), por lo que las principales fuentes del río estarán en el área de drenaje del monte.

La cuenca del Jordán constituye un sistema complejo divisible en segmentos de características diferenciadas: la parte superior del rio hasta el lago Tiberiades; el río Yarmuk, que conecta con el Jordán poco después de la salida del lago, y la parte inferior del río hasta la desembocadura en el mar Muerto.

El alto Jordán se alimenta principalmente de un grupo de fuentes cársticas que forman los afluentes Hasbani, Dan y Banias. Los tres confluyen en territorio de Istael $]^{4}$. El Dan es el más importante de los tres por su caudal y por ser el menos variable: de 173 a 285 millones de metros cúbicos al año (Mmc) según la época y con una media de $245 \mathrm{Mmc}^{5}$. El Dan nace y fluye totalmente dentro de territorio israelí, pero tiene una cuenca de drenaje muy

3. El actual proceso de paz entre áxabes e israelfes tuvo su inicio en la Conferencia de Madrid el 30 de occubre de 1991, pero el verdadero impulso llegó con la Declaración de Principios firmada por Yasser Arafat y Yitzhak Rabin en Washington el 13 de setiembre de 1993, y con el Tratado de Paz entre Istael y Jordania fitmado el 26 de octubre de 1994.

4. Las referencias a las fronteras de lsrael siempre se harán según las líneas de separación de fuerzas de 1949. Se ha cscogido esta opción porque es la más aceptada internacionalmente después de las resoluciones 242 y 338 del Consejo de Seguridad de las Naciones Unidas.

5. Los datos disponibles sobre caudales y consumo de agua son muy variables y poco fiables debido a la irregularidad de los caudales, a la dificultad de las mediciones por impedimencos políticos y a la poca confianza que ofrecen algunas fuentes también por razones políticas. 
pequeña $-24 \mathrm{~km}^{2}$ - que no permite explicar su volumen. Así, algún autor señala la posibilidad de que la fuente que lo alimenta esté en un depósito subterráneo situado entre la cordillera del Hermon y el valle del Litani, en tetritorio libanés (Kolars, 1992: 24).

La delimitación de las zonas de donde proviene el agua es importante para las posibles conversaciones sobre el reparto del caudal del Jordán. El Dan, al ser el afluente principal del alto Jordán será una pieza central en la discusión. Podemos ver, pues, que no sólo los datos sobre caudales se convierten en cartas a jugar en la mesa de negociación, el conocimiento del terreno y la posibilidad de estudiarlo también lo son. En un contexto competitivo y conflictivo como el de Oriente Medio la "guerra del agua" tiene su primera manifestación en el plano de la información. No se trata sólo de la transmisión de datos poco precisos, sino que cada actor crea un "corpus científicon en el cual apoya sus tesis y pretensiones, o impide a los otros que lo puedan crear (Picard, 1992: 5). La ocupación del su.r del Líbano por parte del ejercito israelí y la prohibición de acceso a algunas zonas, o la Orden Militar $92-15$ de agosto de 1967- que impide el acceso a los datos hidrológicos a los palestinos de los Territorios Ocupados, son ejemplos de esta "guerra" científica e informativa.

El Hasbani tiene la mayor parte de su recorrido en el Líbano y es más irregular que el Dan: varía entre $52 \mathrm{Mmc}$ y $236 \mathrm{Mmc}$, con una media de 138 Mmc. El Banias desciende del rorte de los Altos del Golán (sur del Hermon) —pertenecientes a Siria y ocupados por Israel en junio de 1967-, y varía entre $63 \mathrm{Mmc}$ y $190 \mathrm{Mmc}$, con una media de $117 \mathrm{Mmc}$. El Jordán fluye a través del valle de Hulah para desembocar en el lago Tiberiades.

La principal salida de agua del lago es el Acueducto Nacional, que transporta el agua hasta el desierto del Neguev, con un caudal que enla década de 1980 fue de entre $420 \mathrm{Mmc}$ y $500 \mathrm{Mmc}$, pero que varía según el nivel del lago. Hacia el Jordán, en la actualidad, sólo salen pequeñas cantidades de agua muy salina o residual (Kliot, 1994: 181,214), cosa que agrava el problema de la salinidad del agua del Jordán inferior.

El río Yarmuk nace en Siria para pasar posteriormente a marcar la frontera entre este país y Jordania durante $40 \mathrm{~km}$, y entre Israel y Jordania en un corto trecho, hasta desembocar en el Jordán. El Yarmuk tiene un caudal de entre $450 \mathrm{Mmc}$ y $500 \mathrm{Mmc}$ alimentado sólo por las cuencas de drenaje siria y jordana. El Jordán, después de la confluencia con el Yarmuk, señala la frontera entre Israel y Jordania durante $40 \mathrm{~km}$ y posteriormente, hasta el mar Muerto, separa la Cisjordania de Jordania. El agua del curso inferior del río es extremadamente salina, hasta el punto de ser inutilizable.

\section{El inicio del conflicto}

Las fronteras (del Hogar Nacional Judio) no deberían ser trazadas exclusivamente sobre los límites históricos (bíblicos) [...] Nuestras pretensiones hacia el norte están imperativamente dictadas por las necesidades de la vida económica moderna $[\ldots]$ Todo el futuro económico de Palestina depende de su 
aprovisionamiento de agua para el regadío y para la producción de electricidad (Chaïm Weizmann, 1919) ${ }^{6}$.

Esta carta de Weizmann, escrita en unos momentos decisivos para la historia de Oriente Medio, no era un hecho aislado ni inusual en las relaciones de los británicos con el movimiento sionista. Pocos años más tarde, y en el mismo contexto histórico, Weizmann, en 1922, diría ante la Conferencia de Paz de París que los sionistas pretendían crear en Palestina un Estado "que había de ser tan judio como Inglaterra inglesas (Benz y Graml, 1987: 145). Para conseguir esta "juevización" la ocupación de la tierra era un primer paso esencial y debía ser real y efectiva. Así, la agricultura pasaba a ser un objetivo primordial en la estrategia sionista con dos intenciones: poseer y controlar físicamente el territorio, y asegurar la autosuficiencia alimentaria. Este objetivo implicaba asegurar el aprovisionamiento de agua.

La cooperación entre los británicos y el movimiento sionista es muy anterior a la Primera Guerra Mundial. En el año 1867 la Palestine Exploration Fund envió un grupo de expertos e ingenieros para estudiar los recursos naturales de la zona, incluyendo el agua. El informe que publicaron en 1871 contenía la primera referencia a la posibilidad de que Palestina y el Neguev absorbieran una inmigración de millones de personas desviando agua del norte de Palestina hacia el sur (Kahhaleh, 1981: 9). Otros estudios posteriores señalaron la posibilidad de desviar agua del Nilo hacia el Sinaf́ (Riyadh, 1985: 10), con lo que ya quedaba claro que el agua era un elemento clave para la futura colonización judía y que las opciones eran pocas: el norte de Palestina con las fuentes del Jordán, el Litani o el Nilo. Por esta razón Weizmann extendía las dermandas territoriales sionistas hasta incluir el Litani y el Yarmuk con sus respectivas cuencas.

La colonización judía, iniciada bajo el dominio otomano, recibió un fuerte impulso con el mandato británico. Entre 1922 y 1947 la población judia de Palestina creció de 84.000 (sobre 668.000 árabes) a 600.000 (sobre 1.230 .000 árabes). El aumento de la población no supuso sólo una mayor presión sobre la tierra y la vida económica, también lo fue sobre los recursos hidrológicos. Muy pronto las estimaciones de las necesidades de agua de la población local, en oposición a las de los recién llegados, se convirtieron en una cuestión politica en el debate sobre la inmigración en Palestina (Naff y Matson, 1984: 30).

El debate se centró en una serie de proyectos técnicos preparados por las distintas partes, los más importantes de los cuales fueron el Plan Ionides -ingeniero británico que trabajaba para el gobierno de Transjordania - para los jordanos, y el Plan Lowdermilk para los sionistas. Mientras que el primero negaba que hubiera suficientes recursos para mantener un nuevo Estado basado en la inmigración, el segundo afirmaba que había bastante agua para

6. Carra escrita en 1919 por Chaïm Weizmann, presidente de la Organización Sionista Mundiat y futuro primer presidente de Israel, y dirigida al primer ministro británico, David Lloyd George (citada por Chesnot, 1993: 167). 
cuatro millones más de inmigrantes judíos. La diferencia estaba en un elemento claramente desestabilizador del Plan Lowdermilk: la utilización del Litani, un tío que no forma parte de la cuenca del Jordán y que no es internacional, pues nace y muere dentro del Líbano.

Paralelamente al debate continuaron las presiones sobre las autoridades mandatarias. La estrategia sionista se planteó dos objetivos esenciales: obtener concesiones para explotar recursos en el futuro e impedir el acceso de los árabes a recursos que afectaran a sus proyectos. Estas presiones dieron resultado en concesiones británicas sobre la explotación de los ríos Yarkon y Kishon (Awja y Muqatta); la desecación de los pantanos del valle de Hulah, y la "concesión Rothenberg" ${ }^{7}$ (Kahhaleh, 1981: 10, 11). Todas estas concesiones suponían una agresión a los derechos de la población árabe.

\section{9: la cuestión del agua en la posguerra}

Después de la primera guerra árabe-israelí, que supuso la partición de hecho del territorio de Palestina entre Israel, Transjordania y Egipto, en unas condiciones mucho más favorables para Israel que las del Plan de Partición de las Naciones Unidas, las negociaciones de los Acuerdos de Armisticio de 1949 no trataron el problema del agua.

Siria y Líbano no tenían un problema inmediato de carestía ni necesidad de los recursos del Jordán, pero para la población israelí, jordana y palestina la situación era muy distinta.

Transjordania, que al año siguiente se fusionaría con Cisjordania para crear el Reino Hashimita de Jordania, tuvo que afrontar uno de los momentos más críticos de su economía. Alrededor de 450.000 palestinos expulsados de Israel se refugiaron en el territorio jordano. Al ser la mayoría de los refugiados campesinos y jornaleros la agricultura fue la única vía para ocuparlos que no exigía una inversión desmesurada en nueva formación profesional y en bienes de equipo. El desarrollo agrícola se convirtio en un objetivo central de la política económica jordana a pesar de que las perspectivas no era prometedoras. $\mathrm{La}$ agricultura jordana era básicamente de secano y el terreno con lluvia suficiente para el cultivo muy limitado: la transición al regadio se imponía e, imprescindiblemente, Ia búsquteda de nuevas fuentes de agua (Naff y Matson, 1984: 33-35).

El nuevo Estado de Israel se encontró con diversos problemas relacionados con el agua desde el mismo momento de su creación. Buena parte de su territorio quedaba fuera de la cuenca del Jordán y con gran carestía de recur-

7. Rothenberg era el director de la Palestine Electric Corporation que recibió la concesión sobre el uso del agua del Yarmuk para generar energfa eléctrica. Así, un judío pasaba a conrolar la principal fuente que alimentaba la Transjordania y la Cisjordania, zonas sin prácticamente población judia, dándole el poder de veto sobre su desarrołlo fucuro. La concesión aún es más pecaliar si se tiene en cuenta que quien la concedí, Sir Herbert Samuel, más tarde fue el mismo presidente de la Palesine Electric Corporation (Arab Palestine Office, 1954: 19). 
sos hidrológicos. A pesar de que la población total de Israel no $\mathrm{creció}^{8}$, las costumbres de los inmigrantes europeos eran mucho más consumidoras de agua que las de la población indígena, sobre todo en lo referente a la agricultura, cosa que incrementó la presión sobre los recursos.

La agricultura fue una de las bases del nuevo Estado a nivel ideológico, demográfico, económico y de seguridad: las colonias agrícolas eran la mejor forma de consolidar la población en áreas dispersas y de crear zonas defendidas. Así, el desarrollo agrícola durante el primer decenio de Israel tenía tres objetivos principales: la absorción de la inmigración judía, la colonización efectiva del territorio con la expułsión de la población palestina y la producción de adimentos (Davis, 1985: 18). Sin embargo, la nueva agricultura israelí era más intensiva en agua que la agricultura tradicional de la zona y, evidentemente, también más que el desarrollo del sector industrial. Se puede apreciar, pues, que la escasez de recursos hidrológicos no fue un elemento determinante en el momento de definir el modelo de desarrollo y de consolidación del nuevo Estado de Israel. Por esta razón el problema del agua crecería en importancia sin que se pudiesen encontrar soluciones basadas en la limitación del consumo.

Las necesidades de Israel y de Jordania y la precariedad del armisticio los ilevaron a planificar unilateralmente la gestión y el desarrollo de los recursos hidrológicos.

Israel formuló el Plan de Siete Años que integraba todos los ámbitos relacionados con el agua, con un único sistema que coordinaba el suministro a los sectores agrícola, industrial y doméstico. El objetivo primero era incrementar la producción de agua de $810 \mathrm{Mmc}$ a $1730 \mathrm{Mmc}$. Los $920 \mathrm{Mmc}$ suplementarios debían salir de dos fuentes principales: la explotación de las aguas subterráneas, torrentes y fuentes del interior de Isracl $-380 \mathrm{Mmc}-;$ y el río Jordán -540 Mmc- (UNRWA, 1956: 96). El proyecto más importante de este plan era la desviación de agua del Jordán hacia los llanos de la costa mediterránea y el Neguev mediante el Acueducto Nacional (National Water Carrier). Otros elementos del plan fueron el drenaje del valle de Hulah, el sistema regional Galilea-Kishon y la explotación de los depósitos de aguas subterráneas. Esros últimos pronto llegaron al límite y la sobreexplotación creó problemas de salinización.

La pieza central del plan, el Acueducto Nacional, tuvo que esperat por razones financieras y políticas. Jisr Banat Yaqub, el punto donde se debía desviar el agua del Jordán, estaba situado en la zona desmilitarizada entre Israel y Siria. Los israelíes temían que las obras provocaran las protestas sirias y la condena internacional, tai como sucedía con el drenaje de Hulah y la expulsión de los árabes de la zona (Beschorner, 1992: 19). El Acueducto Nacional se convirtio inmediatamente en uno de los principales focos de conflicto entre israelíes y árabes. La desviación del agua del Jordán debía aumentar conside-

8. La exputsión de entre 700.000 y 900.000 palestinos se vio compensada por la inmigración del mismo número de judíos. 
rablemente la salinidad del río e impedir el regadío de cerca de $300 \mathrm{~km}^{2}$ del valle del Jordán: se calcula que el Acueducto ha privado de agua fresca a un total de 40.000 dunums $^{9}$ en Jordania y ha impedido el desarrollo completo de otros 80.000 dunums (Schmida, 1985: 27).

Jordania también inició la planificación del futuro de sus recursos hidrológicos. A principios de los años cincuenta el gobierno jordano y la agencia de las Naciones Unidas dedicada a los refugiados palestinos (UNRWA) colaboraron en proyectos de regadío para desarrollar la agricultura y facilitar el asentamiento de los refugiados. El ańo 1952, el ingeniero americano Max Bunger, que trabajaba para la UNRWA, presentó el proyecto de embalse en el río Yarmuk, en la zona de Magarin, con una capacidad de $480 \mathrm{Mmc}$. El agua del pantano de Maqarin se debía desviar a un segundo embalse en Addasiyah desde donde se canalizaría hacia la orilla oriental del valle del Jordán. Este proyecto tenía que permitir el regadío de 435.000 dunums en Jordania y de 60.000 en Siria, al mismo tiempo que generaría energía eléctrica. El plan Bunger tenía el objetivo de facilitar el asentamiento de 100.000 refugiados.

El plan Bunger tenía otro factor positivo desde el punto de vista político: permitía utilizar el agua del río Yarmuk sin tener que desviarla al lago Tiberiades -controlado por los israelíes-, y así evitaba la dependencia jordana de Israel. El elemento negativo era que el Tiberiades era una opción más razonable a un nivel puramente técnico y económico, hasta el punto que Ionides soblo había considerado factible el uso del lago como depósito para el agua del Yarmuk (lonides, 1940: 257).

La financiación del plan Bunger fue asumida por la UNRWA y la USTCA (United States Technical Cooperation Agency) en colaboración con el gobierno jordano. En junio de 1953, Siria y Jordania llegaron a un acuerdo sobre el reparto de las aguas del Yarmuk basado en el mismo plan. Israel respondió protestando porque el plan no reconocía sus derechos ${ }^{\text {to }}$. A causa de las protestas israelíes los Estados Unidos retiraron su apoyo financiero al proyecto y presionaron a la UNRWA para que hiciera lo mismo. El plan Bunger quedó aparcado y al mismo Bunger se le destinó al Brasil, pues su presencia en Oriente Medio ponía en dificultades la política norteamericana en la zona y entraba en contradicción con las gestiones de Eric Johnston, que acababa de ser enviado por el presidente Eisenhower para negociar un acuerdo regional sobre el agua.

\section{Las negociaciones Johnston (1953-1956)}

La política de los Estados Unidos durante los años cincuenta en Oriente Medio intentó mantener las buenas relaciones con los Estados árabes evitando dis-

9. Dunum $=0,1$ hectárea.

10. Istael sólo tiene el $0,75 \%$ de la cuenca del Yarmuk y no apora nada de agra al río (0\%). Los israelíes también defendieron que la concesión sobre las aguas del Yarmuk pertenecía a la Palestine Electric Corporation (concesion Rothenberg). 
tanciarse de Israel. El objetivo era frenar la penetración soviética en la zona, y los medios que utilizaron fueron la diferenciación de las políticas "coloniales" de Francia y el Reino Unido y la ayuda económica. En la Administración americana las opiniones estaban divididas sobre la forma de afrontar el problema de Oriente Medio: John Foster Dulles, el secretario de Estado, era partidario de presionar para que se llegara a un acuerdo político entre árabes e israelíes; mientras que Harold Stassen, el Director de Operaciones Exteriores, y Eric Johnston eran más favorables a utilizar el factor económico que tan buenos resultados estaba dando en Europa (Kafkafi, 1992: 167).

En realidad, el objetivo inmediato de la Administración de los EUA, presionada por el Congreso, era encontrar una salida a la carga financiera que suponía la aportación americana a la UNRWA para hacer frente a las necesidades de los 800.000 refugiados patestinos que subsistían en el valle del Jordán. La potenciación de la agricultura y, por tanto, la gestión del agua del Jordán eran los medios que tenían que permitir el asentamiento definitivo de los refugiados (Wishart, 1990: 538). Y, siguiendo criterios funcionalistas, la cooperación sobre estos objetivos tenía que facilitar el acuerdo polftico sobre la cuestión de Palestina.

Eric Johnston, el embajador especial enviado por el presidente Eisenhower, llegó a Oriente Medio en unos momentos de gran tensión en relación con los recursos hidrológicos. El Plan Bunger acababa de fracasar y los israelíes acentuaban la presión iniciando las obras de desviación del Jordán en el puente de Banat Yaqub y expuisando a habitantes árabes de la zona, a pesar de que, según los acuerdos de armisticio, ni sirios ni israelíes tenían soberanía sobre este territorio. Aun así, algunos miembros del gobierno de Israel, como Ben Gurion y Moshe Dayan, eran partidarios de empezar los trabajos. Los trabajos tuvieron que detenerse cuando Dulles, que estaba preparando la misión de Johnston, suspendió la ayuda norteamericana a Israel.

Pocos días antes de la llegada de Johnston la tensión subió al máximo con la matanza de Qibya ${ }^{11}$. En el gobierno de Tel Aviv había posiciones enfrentadas sobre cómo afrontar las relaciones con los vecinos árabes: Ben Gurion y Dayan eran partidarios de la vía militar, mientras que Moshe Sharett tenía más confianza en los medios políticos, incluyendo los canales diplomáticos de las Naciones Unidas y de los Estados Unidos.

El primer obstáculo que se tenía que salvar para iniciar las negociaciones eran las diferencias de principios entre las dos partes: Israel había planeado su desarrollo basándose en el desvío de agua fuera de la cuenca natural del Jordán, mientras que los árabes, apoyándose en las prácticas de Derecho más extendidas, se oponían a este desvío. La desconfianza entre las partes era otro factor que no se podía olvidar en el momento de diseñar un plan para el valle del Jordán: ni Israel ni los árabes querían dejar en manos del otro volúmenes importantes de agua que pudieran ser utilizados para hacer futuros chantajes.

11. Un raid israelí, ordenado por Ben Gurion, contra el pueblo de Qjbya se saldó con el asesinato de 60 hombres, mujeres y nifios (Kafkafi, 1992: 170). 
Finalmente, la principal dificultad sería el reparto del agua a explotar por cada una de las partes.

La presentación del Plan Main a los Estados interesados inició la «diplomacia de lanzadoras de Johnston. El documento base de la misión Johnston era un proyecto, encargado por la UNRWA a la Tennessee Valley Authority - Plan Main-, que no fue bien recibido por ninguna de las partes, las cuales prepararon sus propios planes como contrapropuesta.

El Plan Árabe de 1954 mantenía el principio de uso del agua dentro de la cuenca natural del río, pues su desvío perjudicaba a los demás regantes. También rechazaba el desvío del agua del Yarmuk al lago Tiberiades y, evidentemente, la petición israelí de incluir el Litani en el proyecto. La distribución del agua debía corresponderse con la aportación de los Estados: Israel obtendría $200 \mathrm{Mmc}$, Jordania 861 Mmc, Siria 132 Mmc y Líbano $35 \mathrm{Mmc}$ (Naffy Matson, 1980: 40).

Los dirigentes y, sobretodo, la opinión pública árabe tenían claras objeciones políticas. Una muestra de ello la encontramos en la opinión del Arab Jordan Valley Office (1954: 21): “Quué interés pueden tener los árabes en hacer posible y más fácil para Israel la creación de su fururo, cuando creen que el Estado se ha fundado a expensas de los árabes y que cuanto más poderoso crezca y más población tenga, más grande será el peligro para los árabes? [...] Claramente se concluye que bajo la apariencia de un informe puramente técnico el Plan Johnston esconde la realidad de un programa político para una solución parcial sino completa del problema palestino [...] No podemos concebir como los árabes pueden cooperar en un proyecto que no sólo mejoraría las condiciones económicas del millón de judíos que han ocupado sus casas sino que además ayudaría a atraer otro millón para ocupar más».

El rechazo público al Plan crecio: hubo manifestaciones en Jordania y Egipto; en Hebrón 60.000 palestinos se declararon en huelga de hambre; Haj Amin al-Husseini, un prominente religioso y político palestino, distribuyó un influyente panfleto que atacaba el Plan (Saliba, 1968: 107). Los grupos de oposición a los regímenes árabes, sobre todo los islamistas como Haj al-Husseini o los Hermanos Musulmanes ${ }^{12}$, utilizaron el problema del agua para la agitación política amenazando la estabilidad de los gobiernos.

Israel, en su plan -Plan Cotton-, imponía el desvio de agua al Neguev como principio irrenunciable en la negociación, insistía en el uso del Litani y el lago Tiberiades, y rechazaba la supervisión internacional de la distribución y la participación de agencias de las Naciones Unidas. Además lanzaba una nueva iniciativa, la construcción de un canal del Mediterráneo al mar Muerto (Naff y Matson, 1984: 40).

Las posiciones iniciales de las dos partes estaban muy alejadas, pero, tal como se demostró, Johnston era un magnífico negociador: mimaba, adulaba, prometía, firmaba cheques con fecha a la firma del acuerdo, se levantaba de la

12. Movimiento islamista, fundado por el egipcio Hassan al-Banna en 1928 , que se extendió por todo Oriente Medio y que sigue teniendo una gran fuerza e influencia sobre el islanismo árabe. 
mesa y amenazaba. Las negociaciones técnicas avanzaban y las diferencias se redujeron hasta unos mínimos que no eran obstáculo. Sin embargo, en los primeros años del nasserismo que, desde el golpe de estado en Egipto de los Oficiales Libres en 1952, difundía la idea del panarabismo amenazando no sólo a Israel sino también a los retrógrados regimenes monárquicos del Mundo Árabe, Johrston no supo ver lo que las dos partes y Dulles habían previsto mucho antes: sin un diálogo político previo no habtía acuerdo sobre el agua.

Los principales elementos del Plan resultante de las negociaciones _ Plan Unificado- eran la aceptación del uso del agua fuera de la cuenca según las cuotas acordadas (Jordania $720 \mathrm{Mmc}$, Israel $400 \mathrm{Mmc}$, Siria $132 \mathrm{Mmc}$ y Líbano $35 \mathrm{Mmc}$ ), un embalse del Yarmuk en Maqarin y el uso limitado del Tiberiades, canales en las dos orillas del valle del Jordán inferior para el regadío, y la implicación financiera de los Estados Unidos (Taubenblatt, 1988: 44).

Los gobiernos árabes, en general de acuerdo a nivel técnico con el Plan Unificado, dejaron la decisión politica en manos de la Liga Arabe ${ }^{13}$. De este modo el Egipto de Gamal Abdel Nasser pasó a jugar un papel esencial. El empeoramiento de las relaciones entre Egipto e Israel y el progresivo distanciamiento de Nasser de los Estados Unidos no favorecía las negociaciones. En 1955 hubo diversos incidentes en la frontera egipcio-israelí y la ejecución de dos espías israelíes en El Cairo. Estos hechos bloquearon los contactos secretos entre Nasser y Sharett y, en febrero del mismo año, Sharett informó al gobierno de Tel Aviv que, ante el más que probable fracaso de las negociaciones, las obras en Banat Yaqub se reemprenderían en abril. Así, el Acueducto Nacional volvf́a a ser el centro de la tensión entre árabes e israelíes. Nasser aseguró al presidente de Siria, Shukri al-Quwatli, que Egipto lucharía contra Israel si después del rechazo árabe al Plan Únificado los israelíes reiniciaban las obras en Jisr Banat Yaqub.

Un elemento exterior que afectó negativamente a las negociaciones fue la firma en 1955 del Pacto de Bagdad ${ }^{14}$, que rompía la esperanza de creación de un sistema defensivo propio de la Liga Árabe y desafiaba la posición dominante de Egipto en el Mundo Árabe. Una consecuencia del Pacto fue el avivamiento de la cuestión palestina y de la tensión con Israel como un factor de unión de los países árabes.

13. La Liga Árabe se fundó en 1945 en El Cairo para reforzar los lazos entre los estados árabes y coordinar su política exterior. Desde 1950 se estableció un pacto de defensa colectiva con vistas, principalmente, al conflicto con Israel. Egipto, por su posición central en el Mundo Árabe, por su capacidad militar y por su población, ejetció tin papel de liderazgo que se vio reforzado en la época naserista gracias a la influencia del mensaje panarabista en la opinión árabe. A partir de 1973, después de la guerra del Yom Kippur y de la firma de los Acuerdos de Camp David entre Egipro e Israel, en 1978, el pals del Nilo abandona este rol central en la política árabe para replegarse en sí mismo.

14. El afio 1955 se creó en Bagdad́ la Organización del Tatado Cencral (CENTO), que reunúa al Reino Unido, Irán, Pakistán, Turquía e Iraq para colaborar en la solución de problemas militares y políticos. En 1959 los Estados Unidos se adherieron parcialmente por medio de acuerdos bilaterales de seguridad y en 1961 se nombró a un jefe de Estado Mayor norteamericano. 
El ataque conjunto de Israel, Francia y el Reino Unido contra Egipto, el 29 de octubre de $1956^{15}$, supuso el fracaso definitivo de la misión Johnston en Oriente Medio.

La negociación Johnston puso de manifiesto que el conflicto árabe-istaelí necesitaba una aproximación política o, dicho a la inversa, que una solución técnica de los problemas del agua y de los refugiados no eta posible sin un primer acuerdo a nivel político. También dejó claro que la cuestión del agua constitúa un elemento central en la estrategia de seguridad del Estado de Israel y, por tanto, también sería un factor básico en su futura estrategia militar.

Sin embargo, las negociaciones fueron productivas en la estricta gestión de los recursos hidrológicos durante unos años, pues Israel, Jordania y Siria respetaron unilateralmente las cuotas de agua acordadas en el Plan Unificado, y los Estados Unidos lo utilizaron como criterio básico para decidir las ayudas a conceder para el desarrollo de las infraestructuras hidrológicas en la región.

\section{De la gestión unilateral a la conquista israelí del agua (1955-1978)}

El fracaso en la negociación abrió el camino a la gestión unilateral de los recursos hidrológicos. Los dos principales proyectos que estaban sobre la mesa eran el Proyecto del Gran Yarmuk - jordano y sirio-y el Acueducto Nacional -israelí-.

La gestión de los recursos del Yarmuk se basó desde el inicio en la cooperación de sirios y jordanos. El Proyecto del Gran Yarmuk, inspirado en el Plan Bunger, se dividió en tres fases: la primera era un programa de regadio en la región de al-Muzeirib, en Siria, que debía facilitar el control de las crecidas de invierno y regar en verano; la segunda era el canal del Ghor Este (orilla del Jordán inferior), que se inició en 1958, con ayuda financiera de los Estados Unidos, y se completó en 1964, y la tercera fase consistía en la construcción de los embalses de Maqarin y Mukheiba, que nunca se pudieron realizar debido a las presiones israelies y, aún hoy, son el principal proyecto sobre el que se basa la planificación de los recursos hidrológicos jordanos en el futuro.

La construcción del Acueducto Nacional israelí estaba pendiente del problema de Jisr Banat Yaqub. El año 1954, en el Consejo de Seguridad de las Naciones Unidas, la URSS había vetado el permiso de iniciar las obras en este lugar porque estaba situado en la zona desmilitarizada entre Israel y Siria sobre la cual ninguno de los dos Estados tenía soberanía según los acuerdos de armisticio. Los EUA concedieron ayuda financiera para el desarrollo de las infraes-

15. Ia decisión de Nasser, en julio de 1956, de nacionalizar el canal de Suez provocó un ataque conjunto de franceses, británicos e israelies en el mes de octubre del mismo año, que fue condenado por la ONU, los Estados Unidos y la URSS. Las tropas atacantes se retiraron ante la amenaza soviética de intervención, lo que aumento ef prestigio de la URSS ante los árabes y permitió su mayor implicación en la polísica de Oriente Medio. 
tructuras hidrológicas a condición de que Israel esperara dos años antes de reemprender las obras en Jisr Banat Yaqub. Desde el punto de vista del gobietno israelí el Acueducto era un elemento fundamental para el nuevo Estado, indispensable para las colonias del Neguev - la frontera vecina de Egipto y la más desprotegida-., y se debía realizar a cualquier precio. También eran conscientes de las implicaciones políticas y de seguridad del proyecto, hasta el punto que las decisiones importantes se preparaban en un comité especial del gabinete en el que parricipaban los ministerios de Exteriores, Defensa, Finanzas y Agricultura (Reguer, 1993: 71).

La decisión crucial, ante los problemas que suponian las obras en la zona desmilitarizada, fue la de cambiar el punto de desvío del agua al lago Tiberiades, dentro de las fronteras de Israel. Esto implicaba grandes inconvenientes técnicos: menor calidad del agua y, sobre todo, la diferencia de altitud pues el lago está situado a $-213 \mathrm{~m}$ por debajo del nivel del mar, lo que obliga a un fuerte consumo energético ( $8 \%$ de la producción anual de electricidad de Israel) para bombear el agua hasta $+44 \mathrm{~m}$ sobre el nivel del mar y trasladarla $200 \mathrm{~km}$ al sur (Kliot, 1993: 214).

La construcción del Acueducto Nacional fue respondida por los árabes con un proyecto de desvio del agua de los afluentes superiores del Jordán aprobado por la Primera Cumbre de la Liga Árabe, reunida especialmente para estudiar el problema del agua del Jordán. Las obras de desvío de los ríos Hasbani y Banias se iniciaron en 1964 . La respuesta israelí, ante la amenaza que suponían para el Acueducto que habría quedado sin agua suficiente y de mala calidad, fue militar. Entre 1964 y 1967 los incidentes fronterizos y los ataques israelíes a las obras se repitieron y crecieron en violencia paralizando los trabajos.

La reacción árabe se limitó a protestas en las Naciones Unidas que, evidentemente, no frenaron al gobierno israeli que ya estaba preparando la guerra de junio de 1967 ante el aumento de la tensión con Egipto, la carrera de armamentos y las primeras incursiones guerrilleras de los palestinos. Antes de la guerra el proyecto árabe de desvío de las fuentes del Jordán ya estaba suspendido de forma permanente, por lo que no fue una causa directa del ataque israelí. Sin embargo, sí se puede creer que el control del agua a largo plazo fue uno de los objetivos de la estrategia militar israelí.

La guerra de junio de 1967 dio a Israel el dominio de los Altos del Golán, la Cisjordania, Gaza y el Sinaí. Con el Golán se conquistó también el río Banias y se obtuvo una posición estratégica sobre el Yarmuk. Con la Cisjordania se conseguían las aguas subterráneas de esta zona. El mismo junio de 1967 , con la Orden Militar $92^{16}$, Israel pasaba a controlar el agua de los Territorios Ocupados (Benantar, 1993: 88). El control israelí del Jordán superior se completó en 1978 con la primera invasión israelí del sur del Lfbano y la ocupa-

16. Orden de la autoridad militat israeli de ocupación, del 15 de junio de 1967 , que pone bajo su control todos los recursos hidrológicos de los Territorios Ocupados e impide, incluso, el acceso de los palestinos a los clatos sobre el agta. 
ción de una franja en la frontera de los dos países ${ }^{17}$. La segunda invasión, en 1982, consolidaría la ocupación de esta zona en la que se encuentran las fuentes del río Jordán.

El volumen total de las aguas árabes capturadas entre 1967 y 1978 es de $600-700 \mathrm{Mmc}$ y representa el $40 \%$ del total del agua consumida en Israel. Este volumen es equivalente al aumento del consumo israelí desde mitad de los affos sesenta, de lo que se desprende que la expansión de las colonias y de la producción agricola se ha hecho con agua del Jordán superior y de la Cisjordania (Stauffer, 1985: 77). El control de los recursos hidrológicos se puede considerar que es el beneficio más importante que la ocupación de la Cisjordania, el Golán y el sur del Líbano ha procurado a Israel. Esto nos muestra la importancia del agua en la definición de la estrategia militar y de ocupación de los gobiernos israelies.

\section{Apuntes sobre el presente}

La situación actual ante el problema de los recursos hidrológicos ha dado un giro de 180 grados. La causa principal del fracaso de las negociaciones Johnston a mediados de los años cincuenta - la necesidad de un acuerdo político previo- ya no es un obstáculo en lo que se refiere a las relaciones de Israel con los palestinos y jordanos, al haberse firmado la Declaración de Principios de Washington con los primeros y el Tratado de Paz de Araba con los segundos. Además, la lógica de la negociación es otra muy distinta: el agua ya no tiene el valor político que tenía en los años cincuenta y sesenta, pues Israel está consolidado, la inmigración judía ya no depende de la agricultura y los árabes han reconocido el derecho a la existencia de Israel dentro de las fronteras de 1949.

La posibilidad de un conflicto militar por el agua está cada vez más lejana, pero esto no significa que la cooperación sea una realidad. El diálogo entre las partes es un primer paso positivo, aunque no suficiente pues se están siguiendo las mismas pautas de las últimas etapas de la negociación Johnston: discusión sobre las cuotas de agua a consumir. Además, las conversaciones se basan en los acuerdos técnicos conseguidos por Johnston, que respondían a una realidad y a unas necesidades muy distintas de las acruales, cuando no contrarias $^{18}$.

Actualmente, la lógica de la negociación sobre el agua es más técnica —necesidades, recursos y forma de administrarlos - aún sin perder un cier-

17. El gobierno israell, aprovechando la guterra civil libanesa, se marco el objetivo de debilitar la resistencia palestina asentada en el sur del Libano a través del apoyo a milicias libanesas antipalestinas, junto con los ataques militares de castigo y, finalmente, con las invasiones de 1978 y 1982 y la ocupación de la franja fronteriza, que dio lugar a la resolución 425 del Consejo de Seguridad de las Naciones Unidas que pedia la retirada de Israel de esta zona ocupada.

18. Recordemos que la negociación Johnston tenía el objetivo prioritario de fomentar la agricultura y el asentamiento de los refugiados palestinos y de los inmigrantes judíos. 
to valor político, ya que no se dan en un marco aislado sino en un proceso de negociaciones de paz. Las negociaciones deberían seguir el mismo sistema de incentivos exteriores a través de la ayuda internacional que, en un principio, impulsó las conversaciones de palestinos e israelíes. La política de financiar proyectos después del acuerdo ya la utilizó Johnston y sirvió para que Israel y Jordania respetaran durante un tiempo las cuotas acordadas. Sin embargo, esta política y la confianza de los Estados de Oriente Medio en la ayuda exterior se pueden ver afectadas por el retraso de la ayuda a los palestinos, que está creando graves dificultades a Arafat y al proceso de paz en Palestina.

La estrategia israelí en las conversaciones dependerá de cada interlocutor. El objetivo global es convertir la situación presente de dominio de las fuentes, conseguida por la fuerza, en una situación de iure establecida mediante acuerdos. En las negociaciones con Líbano, Siria y los palestinos la táctica será de intercambiar agua por tierra: ceder tierra a cambio de garantías de suministro y control del agua. La negociación con Jordania será distinta, pues Israel no controla el Yarmuk y no podrá boicotear durante más tiempo la construcción del embalse de Maqarin, ya que el Banco Mundial y los Estados Unidos si no ven en Israel la voluntad de llegar a un acuerdo financiarán la obra a pesar de su oposición. Además, a Tel Aviv le interesa que se resuelva rápidamente este asunto, pues esto facilitaría los contactos con Jordania - el rey Hussein tendría algo con que calmar a su opinión pública--, enfrentaría a los jordanos con Siria -los sirios están explotando el Yarmuk por encima del volumen necesario para el embalse- y ayudaría a desplazar las demandas palestinas de agua hacia Jordania - las cuotas Johnston asignaban agua del Yarmuk a la orilla occidental del Jordán--.

La táctica que sigue Israel en las negociaciones es ia de negar la posibilidad de renunciar a ningún volumen de agua —oposición a discutir cuotas de redistribución-, y desviar las conversaciones hacia el aumento del volumen de los recursos, ya sea con medidas técnicas o con importación de agua. El coste de la negociación para Israel es evidente: tener que renunciar a una parte del agua que consume, lo que enfrentaría al gobierno con el poderoso lobby agrícola.

El desarrollo de las negociaciones actuales está conduciendo a una situación contradictoria que se pondrá de manifiesto a medio plazo: los acuerdos bilaterales sobre el agua pueden ayudar a crear la confianza y las interdependencias que faciliten la firma de tratados de paz, pero la gestión de los recursos hidrológicos no se puede limitar a los contactos bilaterales, sino que debe extenderse a toda la cuenca, lo que se verá dificultado por los mecanismos bilaterales actuales ${ }^{19}$. Por otra parte, la negociación de la cuestión del agua en un marco integrado y global, después de la firma de los tratados de paz, facilitaría el contacto multilateral y una mayor eficiencia en la gestión de la cuenca,

19. Es necesario señalar que las conversaciones multilaterales sobre los recursos hidrológicos en Oriente Medio tocan un marco demasiado amplio para ser productivas $\mathrm{y}$, además, ni Siria ni Lúbano participan en ellas. 
pero retardaría el resultado de una forma que ni Jordania ni Palestina podrían soportar.

Finalmente, es necesario señalar que, si bien el agua no es un elemento central de las conversaciones actuales de paz, la solución del conflicto sobre los recursos hidrológicos se impone con toda urgencia, ya que palestinos y jordanos se encuentran en una situación límite de catestía y los israelíes están muy cerca de este límite. Como avisaban las palabras de Butros Ghali o del rey Hussein de Jordania, el agua se está convirtiendo otra vez en una cuestión de seguridad y esto, en Oriente Medio, es una amenaza demasiado real para la paz que debería obligar a todas las partes y a la comunidad internacional a cooperar para un arreglo justo y permanente.

\section{Bibliografía citada}

ARAB JORDAN VALIEY OFFICE (1954). Commentary on Water Development in the Jordan Valley Region. Beirut: Arab Jordan Valley Office.

Beaumont, Peter; Balke, Gerald H.; Wagstaff, Malcolm J. (1976). The Middle East. A Geographical Study. Londres: John Wiley \& Sons.

BENANTAR, Abdennour (1993). "La question de l'eau dans les négotiations arabo-istaéliennes". REP núm. 47.

Benz, Wolfgang; GramL, Hermann (1987). El siglo XXI. Problemas mundiales entre los dos bloques de poder. Madrid: Siglo XXI.

BESCHORNFR, Natasha (1992). "W/ater and Instability in the Middle East». Adelphi Paper, núm. 273.

Chesnot, Chriscian (1993). La Bataille de l'eau au Proche-Orient. 'París: L'Harmattan.

DAVIS, Uri (1985), "Arab Water Resources and Israeli Water Policies», en: FARID, Abdel Majid; SIRrryeH, Hussein (eds.). Israel and Arab Water. Londres: Arab Research Center (Ithaca Press).

FISHER, W.B. (1978). The Middle East. Londres: Methuen.

IONIDES, M.G. (1940). Report on the Water Resources of Transjordan and their Development. Londres: Government of Transjordan and the Crown Agents for the Colonies.

KAFKaFI, Eyal (1992). "Ben-Gurion, Sharett and the Johnston Plan". Studies on Zionism, vol. 13, núm. 2.

KaHHALeH, Subhi (1981). "The Water Problem in Israel and Its Repercussions on the Arab-Israeli Conflictw. IPS Papers, núm. 9.

KuIOT, Nurit (1994). Water resources and conflict in the Middle East. Londres y Nueva York: Routledge.

KOLARS, John (1992). «Les ressources en eau du Liban. Le Litani dans son cadre régional». Monde arabe, Maghreb Machrek, núm. 138.

NAFF, T.; MATSON, Ruth C. (1984). Water in the Middle East. Conflict or Cooperation? Boulder $y$ Londres: Westview Press.

PICARD, Elizabeth (1992), "Les problèmes de l'eau au Moyen-Orient: désinformation, crise de gestion et instrumentalisation politique». Monde arabe Maghreb Machrek, núm. 138.

REguer, Sara (1993). "Controversial Waters: Exploitation of the Jordan River, 19501980». Middle Eastern Studies, vol. 29, núm. 1. 
RrYAOH, Mahmoud (1985). "Israel and the Arab Water in Historical Perspective", en: FARID, Abdel Majid; S:RRIYEH, Hussein (eds.). Israel and Arab Water. Londres: Arab Research Center (Ithaca Press).

SALiBA, Samir N. (1968). The Jordan River Dispute. The Hague: Martinus Nijhoff.

SCHMIDA, Leslie (1985). «Israeli Water Projects and their Repercussions on the ArabIstaeli Conflict", en: FarID, Abdel Majid; SIRRIYEH, Hussein (eds.). Israel and Arab Water. Londres: Arab Research Cenrer (Ithaca Press).

STARR, Joyce (1992). "La guerra que viene en el Tercer Mundo». El Pais - World Media 11-6-1992.

STAUFFER, Thomas (1985). "Arab Waters in Israeli Calculations: The Benefits of War and the Costs of Peacem, en: Farid, Abdel Majid; SIRRIYEH, Hussein (eds.). Israel and Arab Water, Londres: Arab Research Cenrer (Ithaca Press).

TAUBENBLATT, Selig A. (1988). "Jordan River Basin Wazer: A Challenge in the 1990s", en: STARR, J; STOLL, D.C. (eds.). The Politics of Scarcity. Water in the Middle East. Boulder i Londres: Westview Press.

UNRWA (1956). «Special Report on Jordan». Bulletin of Economic Development, núm, 14, Beirut.

WISHART, David M. (1990). «The Breakdown of the Johnston Negotiarions over the Jordan Waters". Middle Eastern Studies, vol. 26, núm. 4. 